\title{
High expression of fibroblast activation protein (FAP) predicts poor outcome in high-grade serous ovarian cancer
}

\author{
Min $\mathrm{Li}^{1 \dagger}$, Xue Cheng ${ }^{2 \dagger}$, Rong Rong ${ }^{3 \dagger}$, Yan Gao ${ }^{4,5}$, Xiuwu Tang ${ }^{1 *}$ (D) and Youguo Chen ${ }^{1 *}$
}

\begin{abstract}
Background: High-grade serous ovarian cancer (HGSOC) is a fatal form of ovarian cancer. Previous studies indicated some potential biomarkers for clinical evaluation of HGSOC prognosis. However, there is a lack of systematic analysis of different expression genes (DEGs) to screen and detect significant biomarkers of HGSOC.

Methods: TCGA database was conducted to analyze relevant genes expression in HGSOC. Outcomes of candidate genes expression, including overall survival (OS) and progression-free survival (PFS), were calculated by Cox regression analysis for hazard rates (HR). Histopathological investigation of the identified genes was carried out in 151 Chinese HGSOC patients to validate gene expression in different stages of HGSOC.

Results: Of all 57,331 genes that were analyzed, FAP was identified as the only novel gene that significantly contributed to both OS and PFS of HGSOC. In addition, FAP had a consistent expression profile between carcinoma-paracarcinoma and early-advanced stages of HGSOC. Immunological tests in paraffin section also confirmed that up-regulation of FAP was present in advanced stage HGSOC patients. Prediction of FAP network association suggested that FN1 could be a potential downstream gene which further influenced HGSOC survival.
\end{abstract}

Conclusions: High-level expression of FAP was associated with poor prognosis of HGSOC via FN1 pathway.

Keywords: Fibroblast activation protein (FAP), The Cancer genome atlas program (TCGA), High-grade serous ovarian cancer (HGSOC), Survival

\section{Background}

Ovarian cancer is one of the major causes of death in females globally. According to 2018 global cancer statistics, 295,414 new cases and 184,799 deaths were reported [1]. In gynecological oncology, ovarian cancer is less prevalent than breast cancer and cervix cancer, however the death rate of $\mathrm{OC}$ is the highest [1]. The most recent 2020 cancer statistics in United States also confirmed that ovarian cancer is the fifth cause of deaths

\footnotetext{
*Correspondence: tangxiuwu1982@yeah.net; chenyouguo@suda.edu.cn ${ }^{+} \mathrm{Min} \mathrm{Li}$, Xue Cheng and Rong Rong contributed equally to this work. 'Department of Gynecology \& Obstetrics, the First Affiliated Hospital of Soochow University, No.188, Shizi Street, Suzhou, Jiangsu Province 215006, China

Full list of author information is available at the end of the article
}

of females $(13,940$ patients, $5 \%$ of total cancer-related death), only trailing by lung \& bronchus cancer, breast cancer, colon \& rectum cancer, and pancreas cancer [2]. According to the NIH Surveillance, Epidemiology, and End Results program (SEER) survival statistics (20092015), 5-year survivorship of ovarian cancer is only $47.6 \%$ [3], which remained virtually unchanged since the last decade [4].

Based on the immunohistological variation, serous ovarian cancer is the most common subtype of ovarian cancer, which could be further categorized as high-grade and low-grade neoplasm according to tumor Federation International of Gynecology and Obstetrics (FIGO) grade $[5,6]$. High-grade serous ovarian cancer (HGSOC)

C C The Author(s). 2020 Open Access This article is licensed under a Creative Commons Attribution 4.0 International License, which permits use, sharing, adaptation, distribution and reproduction in any medium or format, as long as you give appropriate credit to the original author(s) and the source, provide a link to the Creative Commons licence, and indicate if changes were made. The images or other third party material in this article are included in the article's Creative Commons licence, unless indicated otherwise in a credit line to the material. If material is not included in the article's Creative Commons licence and your intended use is not permitted by statutory regulation or exceeds the permitted use, you will need to obtain permission directly from the copyright holder. To view a copy of this licence, visit http://creativecommons.org/licenses/by/4.0/. The Creative Commons Public Domain Dedication waiver (http://creativecommons.org/publicdomain/zero/1.0/) applies to the data made available in this article, unless otherwise stated in a credit line to the data. 
is the most common, aggressive, and fatal type of ovarian cancer. Almost $30 \%$ of patients died within 5 years of diagnosis [7], mainly because of lack of disease-specific symptoms, prominent biomarkers, and effective therapy or targeted drugs [8-10]. Despite sharing some similar histological characters and terminology, high- and lowgrade SOCs are now acknowledged as two different neoplasms [11]. In 2011, the Cancer Genome Atlas (TCGA) program published the genomic and transcriptomic data of ovarian serous carcinoma, which summarized specific features of HGSOC such as TP53 mutation, extensive DNA copy variation, BRCA1/BRCA2 inactive mutation, CCNE1 aberrations, and other survival-related preliminary transcriptional signatures [12-15].

Fibroblast activation protein (FAP), a cell-surface serine protease, emerges as an imperative factor in cancer-associated fibroblasts (CAFs), especially relevant to tumor occurrence and progression. Structurally, FAP consists of a cytoplasmic tail, a single transmembrane domain, and an extracellular domain [16]. FAP is rarely expressed in healthy adult tissues. However, FAP is usually highly upregulated during tissue remodeling events, including cancers or cancer-associated fibroblasts (CAFs) [17-20]. In addition, FAP is considered as a potential biomarker in certain tumor diagnosis and progression due to its protumorigenic specificity in both enzymatic and non-enzymatic manners [21-24].

In this study, we aim to identify potential biomarkers of HGSOC survival from TCGA ovarian cancer cohort bioinformatics data. We analyzed gene expression, clinical and/or demographic information, and targeted strategies for potential HGSOC biomarkers. In addition, we validated our findings by immunohistological investigation of tissues in a group of Chinese HGSOC patients. Results suggest that FAP expression could be an effective biomarker for HGSOC survival, which warrants further investigation as potential intervention of HGSOC.

\section{Methods}

Dynamic protein analysis of TCGA database

Gene expression data (379 cases, Workflow Type: HTSeq-Counts) and clinical information were downloaded by the TCGAbiolink package in R 3.6.0 (https:// www.R-project.org/) from the official TCGA website. After screening the clinical database, 320 patients were included for G3 histologic grade indicating HGSOC. Then, 2 of these patients were excluded because of lack of relevant information. Finally, a total of 318 participants were recruited in our study.

For gene expression analysis, we downloaded the entire 57,331-gene data in serous cystadenocarcinoma from the TCGA RNAseq database. After data cleaning, 19 of 57,331 genes were excluded because of their insufficient expression in total gene expression (expressions of these excluded genes were $<1$ copy in all participants). Then RNA expression data were transformed to $\mathrm{z}$-scores in survival analysis.

\section{Participants recruitment}

This study followed the Declaration of Helsinki and was approved by the Institution of Research Ethics Committee of the First Hospital affiliated to Soochow University. All participants were fully aware of all protocols of this study, and signed up written consent forms to authorize the utilization of their tissues and relevant information.

For the validation set of the findings in HGSOC TCGA database, 151 Chinese Han patients were diagnosed and recruited by the First Hospital affiliated to Soochow University, the Nanjing Maternity and Child Health Care Hospital, and the First Hospital affiliated to the Nanjing Medical University, from January 2013 to May 2019. After surgery, patients' tumor tissues were prepared into the paraffin sections. Their demographic and clinical characteristics were collected as well.

\section{Immunohistochemistry staining (IHC) and IHC score}

IHC was performed on paraffin sections of ovarian cancer tissues to characterize target gene (FAP) expression profile. Detailed steps can be found in our previous study [25]. FAP antibody (\#66562) was purchased from CST company to incubate the preparing sections overnight at $4{ }^{\circ} \mathrm{C}$ for further staining.

Immunohistochemical staining was performed by using the Boster SABC (rabbit IgG)-POD kit (Wuhan, China) with the recommendation of manufacturer. The abovementioned FAP antibody was used to incubate the preparing sections overnight at $4{ }^{\circ} \mathrm{C}$, and $3,3^{\prime}$ - diaminobenzidine was taken to dye for scoring, which was evaluated by two independent and qualified pathologists who were blinded to actual clinical outcomes. IHC scoring was then established as follows. Percentage of positive cells and intensity of staining of FAP antibody were first calculated and then divided into these three major categories: $\leq 3$, negative or weak; $>3$ and $\leq 6$, Moderate; $>6$, strong.

\section{Association network prediction of targeting gene}

Prediction of functional protein association networks of candidate genes was performed by STRING version 11.0 (https://string-db.org/) and Genecard version 4.13 (www. genecards.org). In order to increase prediction accuracy, only common predictions present in both websites were included.

\section{GO and KEGG pathway analysis of host gene}

The gene ontology (GO) functional annotation and KEGG pathway analysis of host genes of polymorphisms were carried out by using the package 'clusterProfiler' in $\mathrm{R}$ (version 4.0.1). 


\section{Statistical analysis}

All statistical analyses were calculated in $R$ 3.6.0. Overall survival (OS) and progression-free survival (PFS) analyses in SOC patients were conducted by Cox regression and the Kaplan-Meier method. Multivariate Cox analysis was applied to identify potential influence of FAP expression on OS and PFS at different clinical stages of HGSOC. Other relevant demographic and clinical characteristics were compared by Student's $t$ test or Wilcoxon test whenever appropriate between the two groups. For immunohistochemical testing in HGSOC patients, the FAP expression score between Stage I + II and Stage III + IV were compared by Student's $t$. $P$ value $<0.05$ was considered as statistical significance in this study.

\section{Results}

Workflow of gene identification in TCGA database

As shown in Fig. 1, there were 304 and 237 genes with different expressions in OS and FPS analysis, respectively. By comparing early stage (Stage I + II) with advanced stage (Stage III + IV) HGSOC, we identified 544 stage-related aberrantly expressed genes in HGSOC patients. After cross-checking OS, FPS and stage-related genes, only FAP and SSC5D were still present. And, FAP was finally included because of its characteristics of typical enzymecatalyzed activity in uniprot database (www.uniprot.org/) and its potential role in HGSOC patient's survival (Fig. 1).
Associations of FAP expression with overall survival (OS) and progression-free survival (PFS)

Cox regression analysis based on FAP expression was performed to generate survival curves in OS and PFS. As shown in Fig. 2a, low FAP expression group showed a significant protective effect on HGSOC prognosis in OS $(P=0.005)$. Longitudinally, low FAP expression group had $91.1 \%$ survival rate in a period of 12 months, compared with $84.4 \%$ in high FAP expression group. Survival rate in 50 months decreased to $31.9 \%$ in low FAP group and $21.4 \%$ in high group, respectively (Table 1). Results of PFS also showed similar patterns to OS between high and low FAP expression groups $(P=0.008$, Fig. $2 b)$.

\section{Expression of FAP in HGSOC patients}

Sections of 151 HGSOC patients' tumor tissues in different stages were stained with FAP-antibody. We identified an increasing trend of FAP expression with respect to severity of cancer stages (Fig. 3). In addition, strong positive FAPstaining generally showed in membranous and cytosolic compartments in HGSOC tissues. Figure $3 \mathrm{~b}$ revealed the total difference scores between early stage HGSOC patients and advanced patients $(P=0.016)$. Additionally, negative FAP was observed in 33 (21.85\%) patients.

\section{Prediction of network influenced by FAP}

Based on STRING and Genecard, prediction of coinfluence genes with FAP and their potential regulating

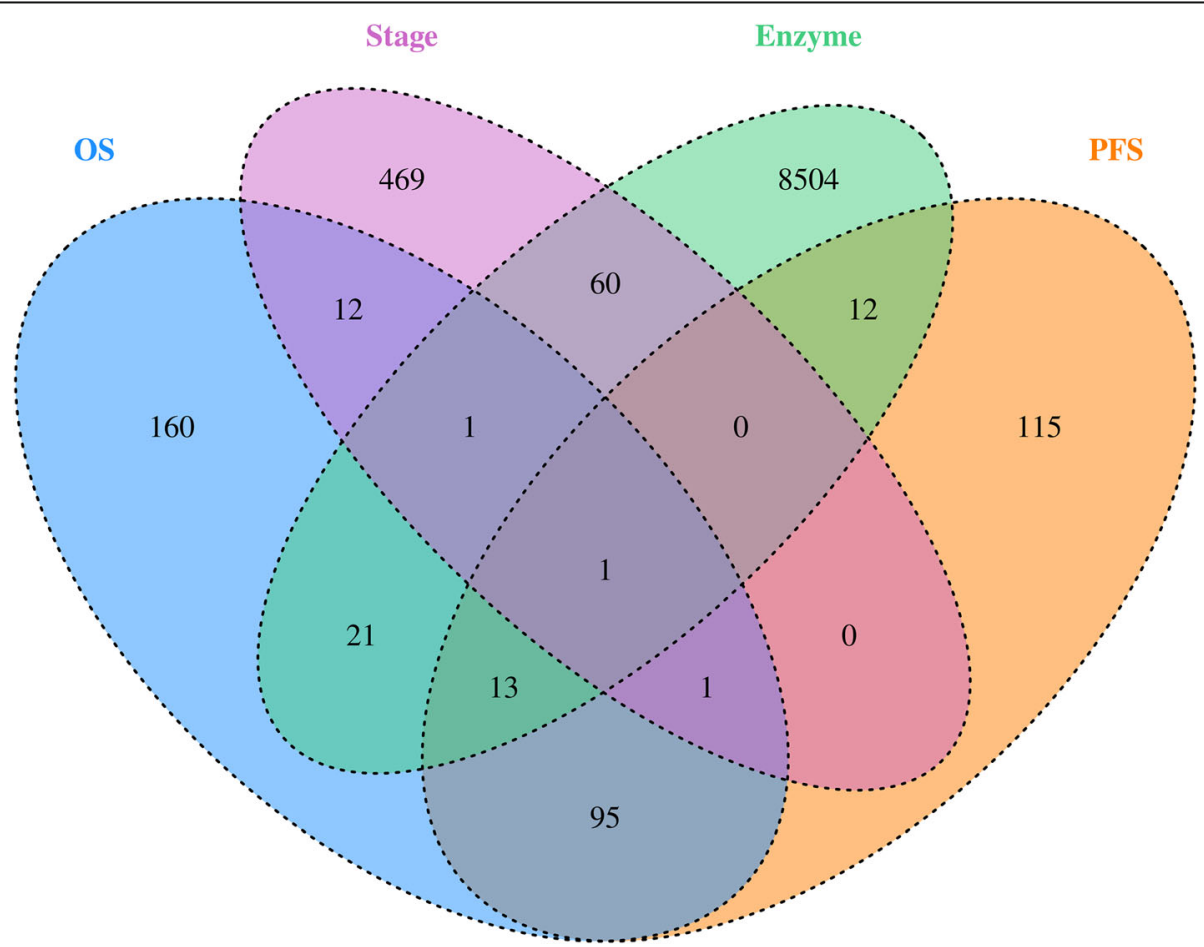

Fig. 1 Venn diagram of target genes selection principle 

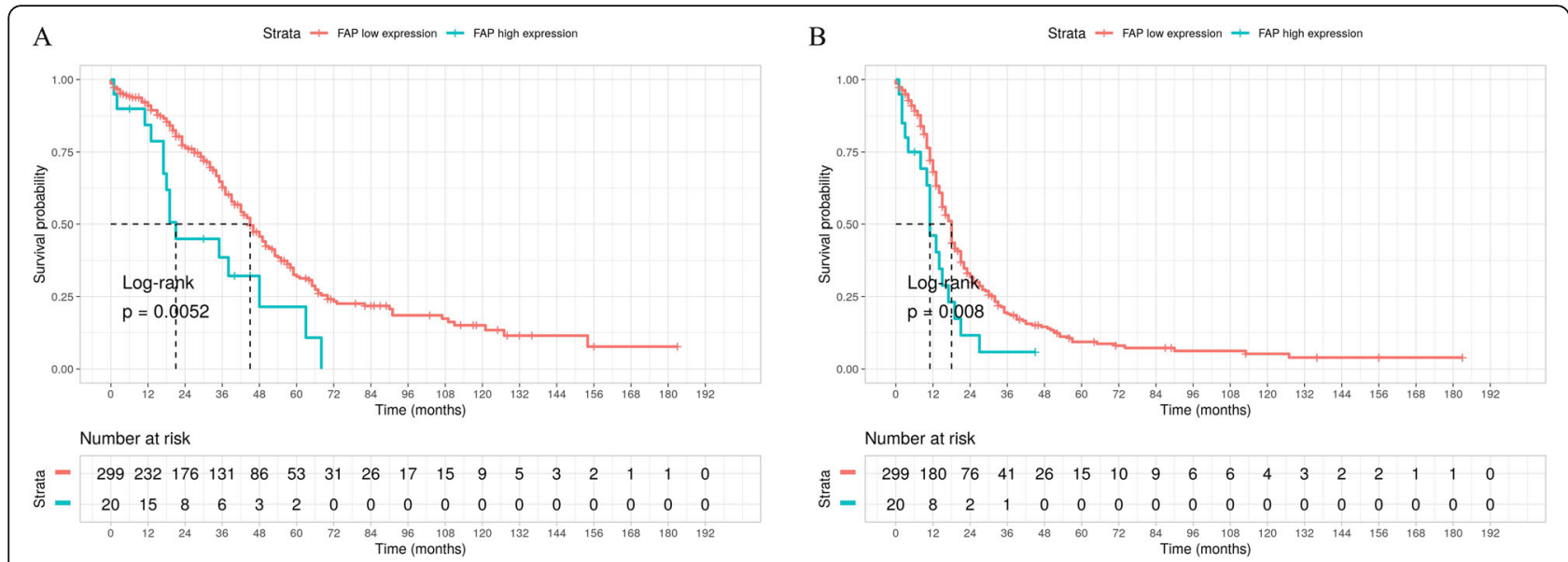

Fig. 2 Impact of FAP expression on survival in HGSOC patients in TCGA cohort. a. Overall Survival, OS; b. Progression Free Survival, PFS

effects was shown in Fig. 4. Specifically, fibronetin-1 (FN1), collagen family genes-COL1A1, COL1A2, COL3A1, COL5A2, Thy-1 cell surface antigen (THY1), and insulin (INS) were identified as co-influence genes.

FN1 was the only significant gene based on Cox regression with its hazardous influence on HGSOC $(P=0.018$, as shown in Table 1). FN1 is demonstrated to overexpress in ovarian cancer, which could eventually influence the formation of multicellular aggregate of ovarian cancer cells, migration and invasion of cancer cells, and aggravating platinum-resistance to deactivate chemotherapy. Additionally, epithelial-mesenchymal transition of ovarian cancer is shown to relate to aberrant expression of FN1. Therefore, FN1 and its regulatory factors, including genes, non-coding RNAs and epigenetic regulations, might be valuable candidates for ovarian cancer studies.

Considering the similar function of collagen (COL) family, we extended the search of collagen encoding genes. Additional COL genes that could influence HGSOC included COL16A1 ( $\mathrm{HR}=2.50, \quad P=0.001$ for Cox regression), COL5A1 $(\mathrm{HR}=2.43, P=0.002)$, COL8A1 $(\mathrm{HR}=2.16, P=$ $0.006)$, and COL4A1 (HR = 1.83, $P=0.035)$ (Table 1$)$.

\section{Bioinformation of FAP in GO and KEGG analysis}

For gene ontology (GO) analysis of FAP, the top three enriched GO annotations were listed as "regulation of fibrinolysis", "negative regulation of extracellular matrix organization", and positive regulation of execution phase of apoptosis" three in Biological Process, while as "dipeptidylpeptidase activity", "aminopeptidase activity", and "metalloendopeptidase activity" in Molecular Function (Fig. 5). On the other hand, however, for KEGG analysis, there is no related available record for FAP in corresponding database.

\section{Discussion}

In this study we extensively searched the TCGA database for HGSOC patients to identify significant biomarkers for HGSOC survival. We constructed a comprehensive summary of differentially expressed genes (DEGs) of HGSOC, especially with regard to patients' survival, in order to evaluate the effects of gene expression on HGSOC survival through data mining.

FAP is a typical plasma membrane-bound serine protease, which is implicated in matrix digestion and invasion [26, 27]. Overexpression of FAP has been investigated and believed to be associated with prognosis in many diseases, especially in cancers [20,22, 28]. Some studies have demonstrated that high FAP expression is a negative prognostic factor for epithelial ovarian cancer [29]. Increased expression of FAP is believed to cause recurrence of epithelial ovarian cancer after chemotherapy $[29,30]$. Considering that HGSOC is a common

Table 1 Significant difference of Predicted gene influenced by FAP in HSOC overall survival COX analysis

\begin{tabular}{|c|c|c|c|c|c|c|}
\hline Ensembl ID & Gene & $\beta$-coefficient & Hazard Rate (HR) & Standard error of coefficient & Z-value & $P$-value \\
\hline ENSG00000078098 & FAP & 0.740663 & 2.097325 & 0.271298 & 2.730071964 & 0.006332 \\
\hline ENSG00000115414 & FN1 & 0.663511 & 1.941597 & 0.28008 & 2.369006093 & 0.017836 \\
\hline ENSG00000084636 & COL16A1 & 0.916471 & 2.500451 & 0.279922 & 3.274028048 & 0.00106 \\
\hline ENSG00000130635 & COL5A1 & 0.887919 & 2.430066 & 0.289441 & 3.067700678 & 0.002157 \\
\hline ENSG00000144810 & COL8A1 & 0.768551 & 2.15664 & 0.279769 & 2.747088883 & 0.006013 \\
\hline ENSG00000187498 & COL4A1 & 0.608281 & 1.837271 & 0.289627 & 2.100225766 & 0.035709 \\
\hline
\end{tabular}




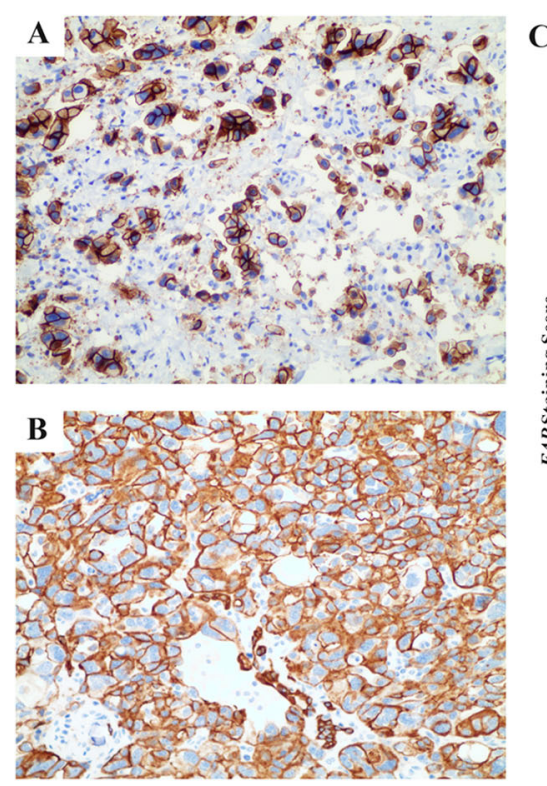

C

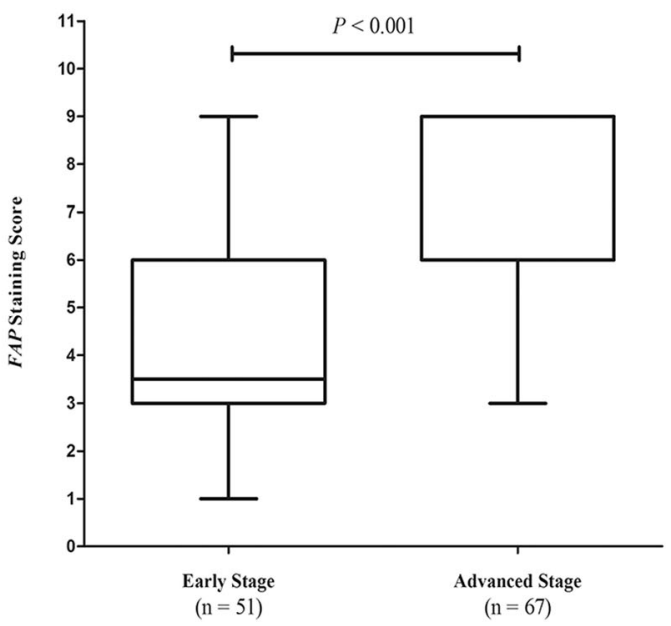

Fig. 3 Expression of FAP in HGSOC tissues. a. Expression of FAP in early-stage patients' tissues. b. Expression of FAP in advanced-stage patients' tissues. c. Total expression of FAP staining in patients with different stages of HGSOC

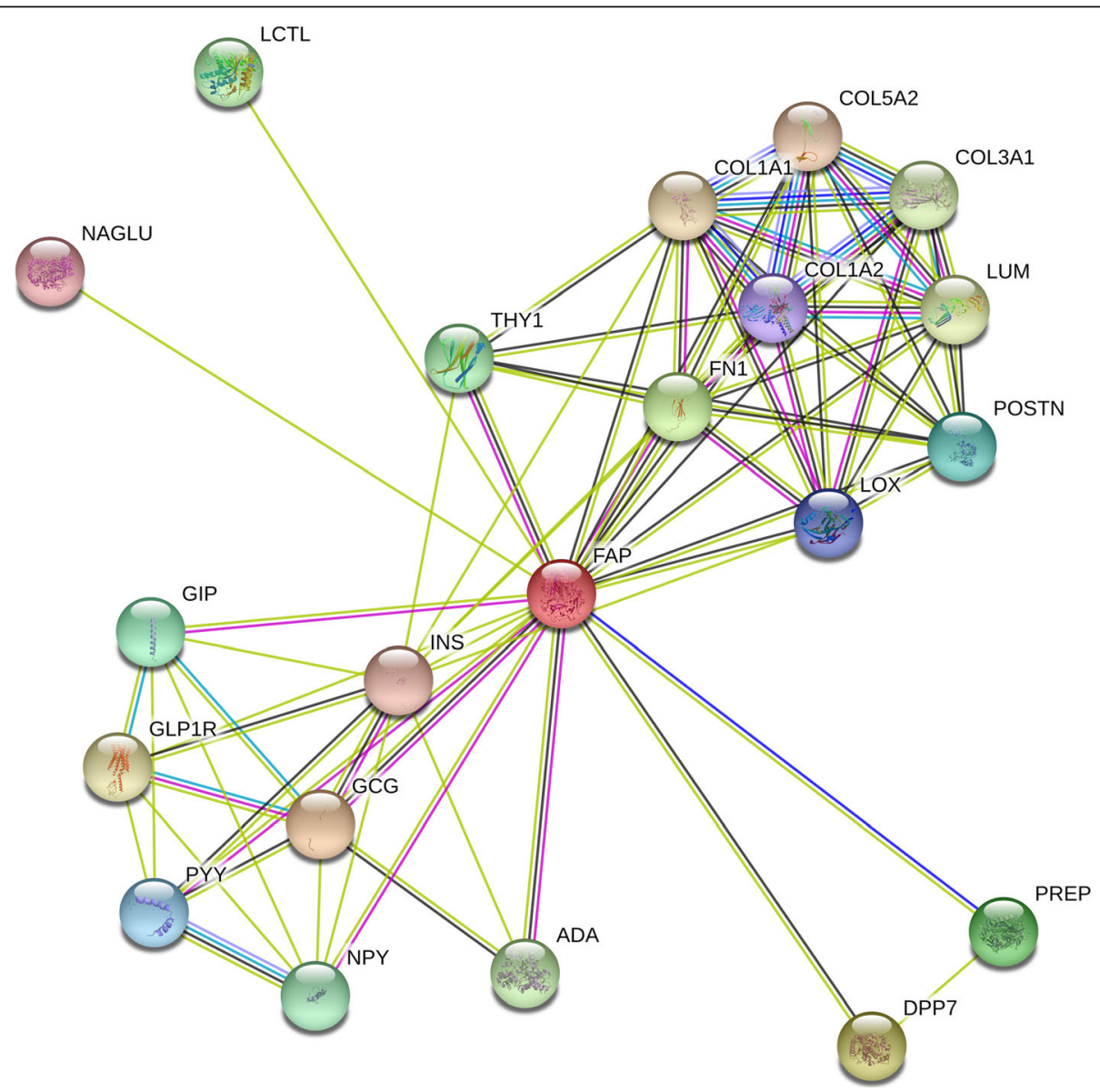

Fig. 4 Prediction of FAP influence in gene regulation 

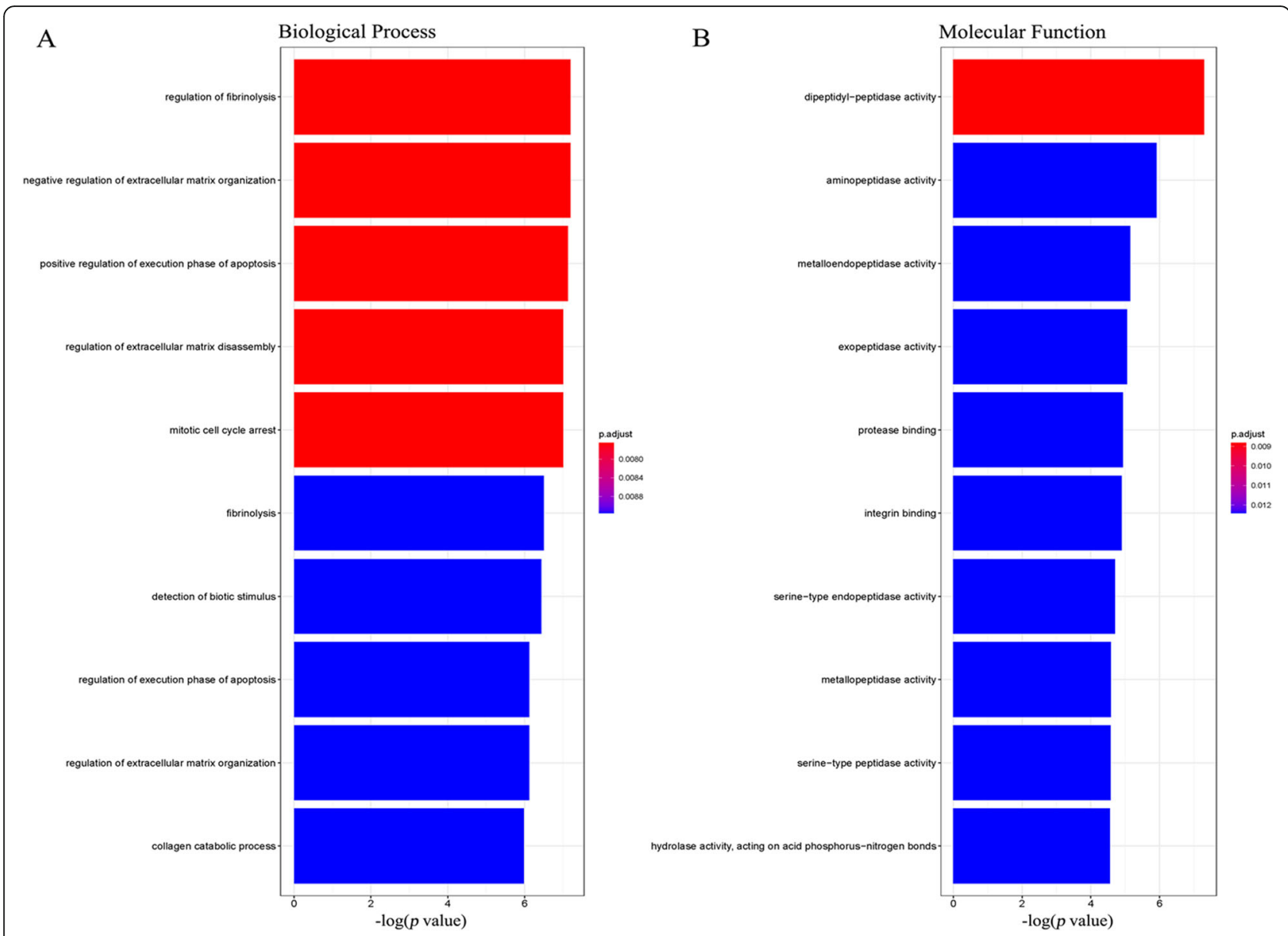

Fig. $5 \mathrm{GO}$ annotations of FAP. a. Biological Process. b. Molecular Function

histological type of epithelial ovarian cancer, our findings in TCGA database on HGSOC support this finding from a new perspective.

Based on the location of FAP in HGSOC tissue, we suggest that FAP could be a strong positive cell-surface receptor. FAP has high expression in HGSOC patients in both Chinese population (over $60 \%$ in our study) and other ethnics (over 50\%) [30]. In addition, performance of therapeutic targeting at FAP also suggests its effectiveness for cancers [31]. Therefore, FAP could be a potential biomarker for drug delivery or even direct therapy for HGSOC.

Cancer-associated fibroblasts (CAFs), including FAP, usually participate in extracellular matrix structure remodeling and tumor microarray reconstruction. In this study, we also retrospectively explored the TCGA database and investigated the effects of other CAF members on HGSOC prognosis, such as ACTA2, PDGFR $\alpha / \beta$, S100A4, and $\alpha$ SMA. These CAF members are widely adopted as biomarkers of HGSOC. Nevertheless, none of these genes showed significant association with HGSOC survival. This result may be partly due to negative expression of $\alpha \mathrm{SMA}$ [30].
We identified $F N 1$ as the only potential gene regulated by FAP in HGSOC. Currently, there is no direct evidence to establish the causal relationship between FAP and FN1. Nevertheless, in this study, we demonstrated similar eventual clinical outcomes induced by $F A P$ and $F N 1$, positive correlation between their expression, significance of their role in overall survival of HGSOC, and direct regulating relationship predicted by STRING and Genecard. All these novel findings suggested $F A P$ as a novel trigger for FN1, at least for HGSOC survival. For THY1 gene, though it was reported as a putative tumor suppressor of ovarian cancer, our study did not produce the same results as previous ones [32], despite THY1's presence in our prediction of potential $F A P$ association networks. It is possible that THY1 cooperates with FAP during HGSOC occurrence but not in prognostic period.

\section{Conclusion}

After extensive data mining of TCGA database, we identified FAP as a significant biomarker for HGSOC 
survival. FAP overexpression led to worse outcome of HGSOC patients, especially in the advanced clinical stage. FN1 expression is potentially down regulated by FAP and further influences HGSOC survival.

\author{
Abbreviations \\ HGSOC: High-grade serous ovarian cancer; DEGs: Different expression genes; \\ OS: Overall survival; PFS: Progression-free survival; HR: Hazard rate; \\ FAP: Fibroblast activation protein alpha; FN1: Fibronectin 1; TCGA: The \\ Cancer Genome Atlas Program; SEER: Surveillance, Epidemiology, and End \\ Results program; FIGO: Federation International of Gynecology and \\ Obstetrics; CAFs: Cancer-associated fibroblasts; IHC: Immunohistochemistry \\ staining; GO: Gene ontology; KEGG: Kyoto Encyclopedia of Genes and \\ Genomes; THY1: Thy-1 cell surface antigen; INS: Insulin
}

\section{Acknowledgements}

We thank the study participants in our study. We also thank the help of Dr. Yan Gao in Suzhou Center for Disease Prevention and Control. And finally, we appreciate the technical support on statistics from Target-Gene biotechnology co. LTD in Nanjing.

\section{Authors' contributions}

XWT and YGC made substantial contribution to design the entire research. ML made substantial acquisition and analysis of corresponding data. XC, RR and YG collected the paraffin-sample of OV patients and performed the $\mathrm{IHC}$ for validation. XWT wrote the article. All authors revised the manuscript and approved the final manuscript for publication.

\section{Funding}

This study was supported by Suzhou industrial technology innovation projection (201900180051, SYS2019041), Jiangsu Maternal and Child Health Association Research Program (FYX202002), and the Jiangsu Provincial Medical Youth Talent (QNRC2016231).

\section{Availability of data and materials}

The main datasets for screening DEGs and analysis during the current study are available from TCGA database. And the corresponding validation IHC data are available from the corresponding authors upon reasonable request.

\section{Ethics approval and consent to participate}

This study followed the Declaration of Helsinki and was approved by the Institution of Research Ethics Committee of the First Hospital affiliated to Soochow University (No.20180912). All participants were fully aware of all protocols of this study, and signed up written consent forms to authorize the utilization of their tissues and relevant information.

\section{Consent for publication}

Not Applicable.

This manuscript does not contain any individual person's data in any form.

\section{Competing interests}

The authors have no conflicts of interest to declare.

\section{Author details}

'Department of Gynecology \& Obstetrics, the First Affiliated Hospital of Soochow University, No.188, Shizi Street, Suzhou, Jiangsu Province 215006, China. ${ }^{2}$ Department of Pathology, Obstetrics and Gynecology Hospital Affiliated to Nanjing Medical University, Nanjing 210004, China. ${ }^{3}$ Department of Pathology, the First Affiliated Hospital with Nanjing Medical University, Nanjing 210029, China. ${ }^{4}$ Institute of Suzhou Biobank, Suzhou Center for Disease Prevention and Control, Suzhou 215004, China. ${ }^{5}$ School of Public Health, Medical College of Soochow University, Suzhou 215123, China.

Received: 10 May 2020 Accepted: 19 October 2020

Published online: 27 October 2020

\section{References}

1. Bray F, Ferlay J, Soerjomataram I, Siegel RL, Torre LA, Jemal A. Global cancer statistics 2018: GLOBOCAN estimates of incidence and mortality worldwide for 36 cancers in 185 countries. CA Cancer J Clin. 2018;68(6):394.
2. Siegel RL, Miller KD, Jemal A. Cancer statistics, 2020. CA Cancer J Clin. 2020;70(1):7.

3. The Surveillance, Epidemiology, and End Results (SEER) Program. Cancer Stat Facts: Ovarian Cancer. https://seer.cancer.gov/statfacts/html/ovary.html. Accessed 8 Mar 2020.

4. Vaughan S, Coward JI, Bast RC Jr, Berchuck A, Berek JS, Brenton JD, et al. Rethinking ovarian cancer: recommendations for improving outcomes. Nat Rev Cancer. 2011;11(10):719.

5. Cho KR, Shih IM. Ovarian cancer. Annu Rev Pathol. 2009;4:287.

6. Silverberg SG. Histopathologic grading of ovarian carcinoma: a review and proposal. Int J Gynecol Pathol. 2000;19(1):7.

7. Montavon C, Stricker GR, Schoetzau A, Heinzelmann-Schwarz V, Jacob F, Fedier A. Outcome in serous ovarian cancer is not associated with LATS expression. J Cancer Res Clin Oncol. 2019;145(11):2737.

8. Cole AL, Austin AE, Hickson RP, Dixon MS, Barber EL. Review of methodological challenges in comparing the effectiveness of neoadjuvant chemotherapy versus primary debulking surgery for advanced ovarian cancer in the United States. Cancer Epidemiol. 2018;55:8.

9. Ozols RF. Challenges for chemotherapy in ovarian cancer. Ann Oncol. 2006; 17(Suppl 5):v181.

10. Bowtell DD, Bohm S, Ahmed AA, Aspuria PJ, Bast RC Jr, Beral V, et al. Rethinking ovarian cancer II: reducing mortality from high-grade serous ovarian cancer. Nat Rev Cancer. 2015;15(11):668.

11. Kurman RJ, Carcangiu ML, Herrington S, Young RH. WHO Classification of Tumours of Female Reproductive Organs. Fourth Edition. IARC WHO Classification of Tumours; 2014. https://www.amazon.com/ClassificationTumours-Female-Reproductive-Medicine/dp/9283224353.

12. Hanahan D, Weinberg RA. Hallmarks of cancer: the next generation. Cell. 2011;144(5):646.

13. Zhang H, Liu T, Zhang Z, Payne SH, Zhang B, McDermott JE, et al. Integrated Proteogenomic characterization of human high-grade serous ovarian Cancer. Cell. 2016;166(3):755.

14. Kaldawy A, Segev Y, Lavie O, Auslender R, Sopik V, Narod SA. Low-grade serous ovarian cancer: a review. Gynecol Oncol. 2016;143(2):433.

15. Cancer Genome Atlas Research N. Comprehensive genomic characterization of squamous cell lung cancers. Nature. 2012;489(7417):519.

16. Aertgeerts K, Levin I, Shi L, Snell GP, Jennings A, Prasad GS, et al. Structural and kinetic analysis of the substrate specificity of human fibroblast activation protein alpha. J Biol Chem. 2005;280(20):19441.

17. Niedermeyer J, Garin-Chesa P, Kriz M, Hilberg F, Mueller E, Bamberger U, et al. Expression of the fibroblast activation protein during mouse embryo development. Int J Dev Biol. 2001;45(2):445.

18. Mankaney G, Leone P, Cruise M, LaGuardia L, O'Malley M, Bhatt A, et al. Gastric cancer in FAP: a concerning rise in incidence. Familial Cancer. 2017; 16(3):371.

19. Yang $X$, Lin Y, Shi Y, Li B, Liu W, Yin W, et al. FAP promotes immunosuppression by Cancer-associated fibroblasts in the tumor microenvironment via STAT3-CCL2 signaling. Cancer Res. 2016;76(14):4124

20. Chen L, Qiu X, Wang X, He J. FAP positive fibroblasts induce immune checkpoint blockade resistance in colorectal cancer via promoting immunosuppression. Biochem Biophys Res Commun. 2017;487(1):8.

21. Hamson EJ, Keane FM, Tholen S, Schilling O, Gorrell MD. Understanding fibroblast activation protein (FAP): substrates, activities, expression and targeting for cancer therapy. Proteomics Clin Appl. 2014;8(5-6):454

22. Dong R, Guo J, Zhang Z, Zhou Y, Hua Y. Polyphyllin I inhibits gastric cancer cell proliferation by downregulating the expression of fibroblast activation protein alpha (FAP) and hepatocyte growth factor (HGF) in cancerassociated fibroblasts. Biochem Biophys Res Commun. 2018;497(4):1129.

23. Ruan P, Tao Z, Tan A. Low expression of miR-30a-5p induced the proliferation and invasion of oral cancer via promoting the expression of FAP. Biosci Rep. 2018;38(1):BSR20171027.

24. Kilvaer TK, Rakaee M, Hellevik T, Ostman A, Strell C, Bremnes RM, et al. Tissue analyses reveal a potential immune-adjuvant function of FAP-1 positive fibroblasts in non-small cell lung cancer. PLoS One. 2018;13(2):e0192157.

25. Gao Y, Tang X, Cao J, Rong R, Yu Z, Liu Y, et al. The effect of HER2 single nucleotide polymorphisms on cervical Cancer susceptibility and survival in a Chinese population. J Cancer. 2019;10(2):378.

26. O'Brien P, O'Connor BF. Seprase: an overview of an important matrix serine protease. Biochim Biophys Acta. 2008;1784(9):1130.

27. Yu DM, Yao TW, Chowdhury S, Nadvi NA, Osborne B, Church WB, et al. The dipeptidyl peptidase IV family in cancer and cell biology. FEBS J. 2010; 277(5):1126 
28. Yu LN, Liu Z, Tian Y, Zhao PP, Hua X. FAP-a and GOLPH3 are hallmarks of DCIS progression to invasive breast Cancer. Front Oncol. 2019;9:1424.

29. Mhawech-Fauceglia P, Yan L, Sharifian M, Ren X, Liu S, Kim G, et al. Stromal expression of fibroblast activation protein alpha (FAP) predicts platinum resistance and shorter recurrence in patients with epithelial ovarian Cancer. Cancer Microenviron. 2015;8(1):23.

30. Mhawech-Fauceglia P, Wang D, Samrao D, Kim G, Lawrenson K, Meneses T, et al. Clinical implications of marker expression of carcinoma-associated fibroblasts (CAFs) in patients with epithelial ovarian carcinoma after treatment with Neoadjuvant chemotherapy. Cancer Microenviron. 2014;7(12):33.

31. Pure $E$, Blomberg R. Pro-tumorigenic roles of fibroblast activation protein in cancer: back to the basics. Oncogene. 2018;37(32):4343.

32. Abeysinghe HR, Cao Q, Xu J, Pollock S, Veyberman Y, Guckert NL, et al. THY1 expression is associated with tumor suppression of human ovarian cancer. Cancer Genet Cytogenet. 2003;143(2):125.

\section{Publisher's Note}

Springer Nature remains neutral with regard to jurisdictional claims in published maps and institutional affiliations.

Ready to submit your research? Choose BMC and benefit from:

- fast, convenient online submission

- thorough peer review by experienced researchers in your field

- rapid publication on acceptance

- support for research data, including large and complex data types

- gold Open Access which fosters wider collaboration and increased citations

- maximum visibility for your research: over $100 \mathrm{M}$ website views per year

At BMC, research is always in progress.

Learn more biomedcentral.com/submissions 